\title{
Retraction Note: A fast, high-affinity fluorescent serotonin biosensor engineered from a tick lipocalin
}

\author{
Shen Zhang (D), Xinyu Li, Shengyu Zhao, Mikhail Drobizhev and Hui-wang Ai® \\ Retraction of: Nature Methods https://doi.org/10.1038/s41592-021-01078-7, published online 25 February 2021
}

We, the authors, are retracting this Brief Communication as some of the original findings could not be reproduced. These reproducibility issues undermine our full confidence in the integrity of the study.

In the initial version of this recently published article, we reported a genetically encoded, fluorescent serotonin biosensor, G-GESS, and the use of G-GESS to image serotonin in cultured cells, brain slices and behaving mice. Regrettably, we, and several other researchers in the corresponding author's laboratory not involved in the original study, have been unable to reproduce some of the reported key results under the conditions originally described.

We recently used the pDisplay-G-GESS-M plasmid to transfect HEK 293T cells and observed that the membrane localization of the biosensor was sensitive to fetal bovine serum (FBS) batches. By using an FBS batch from Gibco (cat. no. 16000-044, lot no. 2103017RP), we were able to replicate membrane localization similar to that reported in Fig. 1e. However, the cells required the addition of several hundred micromolar serotonin for robust fluorescence increase. These new results suggest that the affinity of G-GESS for serotonin is lower than that claimed in the initially published article.

We further used liquid chromatography-mass spectrometry (LC-MS) to analyze the serotonin stock solutions found in our $-80{ }^{\circ} \mathrm{C}$ freezer and observed MS peaks suggesting glutamate contamination in some of the samples. In addition, we used PCR or reverse transcription (RT)-PCR to amplify gene fragments from our viral stocks, and the sequencing results indicate that the viral stocks were contaminated with one encoding a glutamate biosensor.

While we are performing further investigation, in light of the existing findings, we wish to retract the paper.

In the initial publication, the sensor was tested in several cell culture systems, brain slices and behaving mice. We are at present unable to fully explain the observed sensor responses described in the initial article, but it is highly possible that these responses could be caused by the contaminated chemical or viral stocks.

We deeply apologize to the scientific community for not detecting these problems sooner.

All authors agree to this retraction.

Published online: 23 April 2021

https://doi.org/10.1038/s41592-021-01148-w

(c) The Author(s), under exclusive licence to Springer Nature America, Inc. 2021 